\title{
Wiskott Aldrich Syndrome, often Missed: A Case Report and Review
}

\author{
Kumar $\mathbf{M K}^{1}$, Narayan $\mathbf{R}^{2}$ \\ ${ }^{1}$ Dr. Mani Kant Kumar, MBBS, MD. Assistant Professor, ${ }^{2}$ Dr. Raghvendra Narayan, MBBS, MD. Associate Professor. \\ Both from the Department of Paediatrics, Narayan Medical College and Hospital, Jamuhar, Sasaram, Bihar, India.
}

Address for Correspondence: Dr. Mani Kant Kumar, E-mail: manikant7@yahoo.com

\begin{abstract}
Wiskott-Aldrich syndrome is an X-linked recessive disorder characterized by thrombocytopenia, eczema and recurrent infections. We report a 15 month old boy who had presented with lower gastrointestinal bleed, recurrent infections and eczema. Blood picture revealed microthrombocytopenia, high IgA and $\mathrm{IgE}$, and low IgM and Normal IgG levels. A diagnosis of Wiskott-Aldrich Syndrome was made, which was missed by many paediatrician even after prolonged hospital stay before admission in our Institute. The recent progress in understanding of the pathophysiology and treatment are discussed.
\end{abstract}

Key words: Wiskott-Aldrich syndrome, Eczema, Microthrombocytopenia

\section{Introduction}

W iskott-Aldrich Syndrome (WAS) is an X-linked recessive disorder characterized by a triad of thrombocytopenia with decreased mean platelet volume, eczema, and increased susceptibility to pyogenic and opportunistic infections ${ }^{1}$. Immunologically, there is poor antibody response to polysaccharide antigens, progressive decrease in T cell number and function, and a characteristic immunoglobulin profile reflected in low IgM, elevated /normal IgA, elevated lgE and normal/ low lgG levels².

The rapid destruction of abnormally shaped platelets is the primary cause for severe thrombocytopenia in WAS patients ${ }^{3}$. The immunodeficiency in WAS is primarily associated with $\mathrm{T}$ - and B-lymphocyte dysfunction. The protein encoded by the WAS gene (WASP) is a hematopoietic specific regulator of actin nucleation in response to signals arising at the cell membrane ${ }^{4}$. Mutations impairing but not abolishing WASP expression can cause $\mathrm{X}$-linked thrombocytopenia $(\mathrm{XLT})$ and is considered an attenuated form of WAS because it is characterized by low platelet counts with minimal or no immunodeficiency5.

Purpose of reporting this case to increase awareness among paediatrician of developing countries regarding high index of suscpicion and to think other possibilities if any patient not improving to the exetent expected inspite of adequate treatment. Here we are reporting a case with Wiskott-Aldrich syndrome from Bihar, India and recent progress in understanding the pathophysiology of the disease and treatment are discussed.

\section{Case Report}

A 15-month-old boy, born by spontaneous vaginal delivery of a non-consanguineous marriage presented with second episode of bleeding per rectum. There was no history of decreased urine output, no abdominal pain, no swelling over body, no family history of similar illness in family. There was history suggestive of recurrent sinopulmonary and soft tissue infections, eczematoid rashes which was started form scalp and gradually progressed to all over body since early infancy which was treated by many paediatricians. First episode of bleeding per rectum occurred 2 months back, during first episode initially treated by a paediatrician as case of dysentery but no improvement in the bleeding then he referred this case to another hospital, where he was diagnosed as case of Hemolytic Uremic syndrome. Patient was hospitalized for 40 days and managed with 6 times fresh whole blood transfusion, broad spectrum antimicrobials and was discharged on request when 
asymptomatic for 5 days. After 15 days of discharge patient again developed bleeding per rectum then brought to our institute. Investigation during first episode revealed Hemoglobin $7.5 \mathrm{gm} / \mathrm{dl}$, TLC 12400/cmm, DLC$P_{58}, L_{40}, E_{02}$, Platelet count 20,000/cmm, general blood picture (GBP) - microcytic hypochromic anemia, no fragmented RBC, Tiny platelets, no immature cell, blood urea $18 \mathrm{mg} / \mathrm{dl}$, Creatinine $0.6 \mathrm{mg} / \mathrm{dl}$, urine was normal normal on routine examination and microscopy, inspite of 6 times fresh whole blood transfusion predischarged platelet count was $36,000 / \mathrm{cmm}$.

On admission in our hospital he was afebrile, alert and active. He had mild pallor pulse rate 92 per minute, respiratory rate of 32 per minute, weight $5.5 \mathrm{~kg}$ (less than $3^{\text {rd }}$ centile), length $70 \mathrm{~cm}$ (less than $3^{\text {rd }}$ centile), sparse hair and eczematoid rash over the scalp, neck, skin folds of upper and lower limbs. His head circumference was $43 \mathrm{~cm}$ (normal for age) and chest circumference $41 \mathrm{~cm}$. There were no congenital malformations. Respiratory, cardiovascular and central nervous system were within normal limits while the liver was palpable 1 $\mathrm{cm}$ below the costal margin. Laboratory investigations revealed hemoglobin $(\mathrm{Hb})$ of $9.6 \mathrm{gm} / \mathrm{dl}$, total leukocyte count 11,800/cumm and platelet count 25,000/cumm. PT (Prothrombin time) was 13 seconds and aPTT was 31 seconds. General blood picture revealed normocytic hypochromic with microthrombocytopenia. Blood urea was $16 \mathrm{mg} / \mathrm{dl}$ and creatinine was $0.6 \mathrm{mg} / \mathrm{dl}$ and Urine was normal on routine examination and microscopy, urine culture was sterile. Immunoglobulin profile showed high $\lg \mathrm{A}$ (179mg/dl, normal less than $83 \mathrm{mg} / \mathrm{dl}$ for age ), low lgM (95mg/dl, normal >145 mg/dl), High lgE (1560unit/ $\mathrm{ml}$ normal 1.4- 52.3 unit/ml for age) and normal lgG (1378 mg/dl), HIV serology was negative.

Patients was managed with oral antimicrobials, multivitamins, one time@ $20 \mathrm{ml} / \mathrm{kg}$ fresh whole blood transfusions(because platelets concentrates not available in our blood bank) and IV immunoglobulin at rate $400 \mathrm{mg} / \mathrm{kg}$, he improved gradually, and pre discharged platelets count was 55,000/cumm. Parents were counselled regarding available treatments options and after 7 days of hospital stay he was dischared on Co-trimoxazole prophylaxis, multivitamins \& iron supplementation, emmoliants as advised by dermatologist with advice of monthly IV Ig replacement therapy. He was on regular monthly Immunoglobulin replacement therapy in follow up and doing well, and has gained weight $2 \mathrm{~kg}$ over past 5 months.

\section{Discussion}

The present case demonstrated characteristic clinical triad of Wiskott-Aldrich syndrome-intermittent bleeding because of thrombocytopenia, progressive eczema since early infancy and recurrent sinopulmonary bacterial infections. Immunoglobulin profile showed high $\lg \mathrm{A}$, low IgM, High IgE and normal IgG. Small platelets size and low platelets count clinched the diagnosis in favour of Wiskott - Aldrich syndrome. At a later age when $T$ cell functions are affected, opportunistic infections may occur. Children who survive the first 8-10 years are at risk for the development of lymphoid malignancies. The diagnosis of Wiscott Aldrich Syndrome is based on the demonstration of increased serum $\lg A$ and $\lg \mathrm{E}$ and decreased serum IgM levels, absence of isohemagglutinins, and poor anti-body response to polysaccharide antigens in males with characteristic constellation of clinical features ${ }^{2}$. Wiskott-Aldrich syndrome is a complex and severe X-linked disorder characterized by microthrombocytopenia, eczema, immunodeficiency, and increased risk in developing autoimmunity and lymphomas.

WAS affects 1 to 10 of every 1 million male newborns and their life expectancy is approximately 15 years. The protein encoded by the WAS gene (WASP) is a hematopoietic specific regulator of actin nucleation in response to signals arising at the cell membrane $e^{4,5}$. Among clinical manifestations, hemorrhages are frequent ( $80 \%$ incidence) in WAS and range from nonlife-threatening (epistaxis, petechiae, purpura, oral bleeding) to severe manifestations, such as intestinal and intracranial bleeding. Death of WAS patients is caused, in $21 \%$ of the cases, by haemorrhages ${ }^{6}$. Bleeding is the result of severe thrombocytopenia with reduced platelet size, which is the most common finding in WAS and XLT patients (100\% incidence). Thrombocytopenia occurs irrespectively of the severity of the mutation and is possibly caused by instability of mutated WASP in platelets ${ }^{7}$. Despite intensive research, the mechanisms underlying WASP related thrombocytopenia and hemorrhages are not completely understood. Megakaryocyte numbers have been reported to be normal in the majority of WAS patients, whereas proplatelet formation depending on actin polymerization and formation of branching structures is conserved when tested in in vitro and ex vivo cultures ${ }^{8}$. Peripheral destruction of platelets in the spleen is thought to play an important role in thrombocytopenia because a substantial correction of the platelet count and size after splenectomy has been reported. The accelerated destruction could be caused by an intrinsic defect of WASP-deficient platelets, showing an increased surface exposure of phosphatidylserine, or could be mediated by autoimmune reaction because of the presence of antiplatelet antibodies reported in patients and in the murine knockout model ${ }^{9}$. 
The typical skin lesions in WAS patients resemble acute or chronic eczema in appearance and distribution. Eczema develops in $80 \%$ of the patients ${ }^{6}$ and is heterogeneous in severity and persistence. The causes of eczema in WAS patients are currently unknown. WAS patients often have elevated $\operatorname{lgE}$ levels and develop allergies therefore suggesting an atopic origin ${ }^{10}$. Recently, an imbalance in cytokine production toward the Th2 type has been described in WAS patient's T-cell lines and might contribute to the pathogenesis of eczema and allergy ${ }^{11}$. WAS-associated autoimmune complications are frequently observed. The incidence of autoimmunity in classic WAS is high in the US and European populations (40\%-72\%), whereas a lower incidence was reported in Japan $(22 \%)^{6,10,12}$. The most common manifestations are autoimmune hemolytic anemia, cutaneous vasculitis, arthritis, and nephropathy. Less common autoimmune manifestations include inflammatory bowel disease, idiopathic thrombocytopenic purpura, and neutropenia.

Patients frequently have multiple autoimmune manifestation at the same time. Development of autoimmunity have a poor prognosis ${ }^{12}$. Until now, the mechanisms of WAS-associated autoimmunity have not been clarified. It has been proposed that autoimmunity could be the result of a bystander tissue damage originating from the chronic inflammatory state that is established after incomplete pathogen clearance ${ }^{13}$. Moreover, autoimmunity is associated with a higher risk of a later development of tumors and an increased risk of mortality ${ }^{6}$. Two distinct surveys report a tumor incidence of $13 \%{ }^{6}$ and $22 \%{ }^{10}$ in WAS patients. Tumors can arise during childhood (especially myelodysplasia) but are more frequent in adolescents and young adults. WAS-associated tumors are mainly lymphoreticular malignancies, with leukemia, myelodysplasia, and lymphoma (often Epstein-Barr virus [EBV]-positive) resulting in up to $90 \%$ of the cases. WAS-associated malignancies have a poor prognosis because less than $5 \%$ of patients survive 2 years after diagnosis ${ }^{6}$, and result in up to $25 \%$ of death cases ${ }^{13}$. WASP is a key regulator of actin polymerization in hematopoietic cells. As a cytoskeletal regulator, it is necessary for induction of normal immunity. WASp functions as a bridge between signaling and movement of the actin filaments in the cytoskeleton. WASp has several welldefined domains (pleckstrin, cofilin, verprolin, SH3) that are involved in signaling, cell locomotion, and immune synapse formation. In vitro studies with $\mathrm{T}$ cells, platelets, phagocytes, and dendritic cells of patients with Wiskott-Aldrich syndrome reveal defects in the formation of microvilli, filopodia, phagocytic vacuoles, and podosomes, respectively; these structures depend on cytoskeletal reorganization of actin filaments.
Researchers also identified many different mutations that interfere with the protein binding to Cdc42 and Rac GTPases, among other binding partners, most of which are involved in regulation of the actin cytoskeleton of lymphocytes ${ }^{14,15}$. The actin cytoskeleton is responsible for cellular functions, such as growth, endocytosis, exocytosis, and cytokinesis. Mutations of WASP are located throughout the gene and either inhibit or dysregulate normal WASp function. WASp facilitates the nuclear translocation of nuclear factor kappa-B (NF$\mathrm{kB}$ ) and was shown to play an important role in lymphoid development and in the maturation and function of myeloid monocytic cells. In mice, WASp was found to be essential for NF-ATp activation, and for nuclear translocation of p-Erk, Elk1 phosphorylation, and c-fos gene expression in $T$ cells. These defects in mutated forms of WASP are the likely etiology of defective $I L-2$ expression and T-cell proliferation in Wiskott- Aldrich Syndrome.

Research has shown phenotype-genotype correlation. Classic Wiskott-Aldrich syndrome, X-linked thrombocytopenia, and $X$-linked neutropenia occurs when WASp is absent, when mutated WASp is expressed, and when missense mutations occur in the Cdc42-binding site, respectively ${ }^{16}$. Pre-natal diagnosis is possible using DNA technology, either by direct identification of mutation or by linkage.

Treatment is directed mainly at control of bleeding through transfusions of blood and platelets, and control of infections with antibiotics and Immunoglobulin replacement. Splenectomy improves the platelet number but makes the patient susceptible to sepsis, requiring life long prophylaxis with antibiotics ${ }^{17}$. Immunoglobulins given intravnously may be helpful in prophylaxis of both viral and bacterial infections, but do not improve thrombocytopenia. Eczema is difficult to manage and may persist even after long term use of local steroids. Currently, the only curative therapeutic option for WAS patients is hematopoietic stem cell transplantation (HSCT). When a related human leukocyte antigenidentical donor is available, HSCT leads to more than $80 \%$ survival rate. On the other hand, transplantation using the bone marrow of a mismatched related donor results in a decreased survival rate ${ }^{18,19,20,21}$. When a suitable related donor is not available, bone marrow or cord blood transplantation from a matched unrelated donor is a valid therapeutic option, leading to a $71 \%$ to $81 \%$ survival rate ${ }^{19,20,22}$. A better outcome for matched unrelated donor and mismatched related donor HSCT in patients younger than 5 and 2 years of age, respectively, has been reported, suggesting that transplantations should be performed early in life ${ }^{19,20,23}$. 
The first reported case of sucessful BMT for WAS in India from Christian Medical College and Hospital, Vellore $^{24}$. The implementation of new therapeutic strategies, such as transplantation of autologous genecorrected hematopoietic stem cells, is highly desirable because it will avoid rejection and graft-versus-host disease and could be applicable to all WAS patients lacking a suitable bone marrow donor, allowing the timely treatment of the disease. The rationale for gene therapy is also supported by the observation of frequent spontaneous somatic revertants conferring selective advantage to WASP expressing cells ${ }^{25}$. At present, retroviral vectors based on the murine Moloney leukemia virus have been used for the treatment of patients with SCID-X1, ADA-SCID, and X-CGD26,27,28. Recently, a gene therapy trial for WAS was initiated in Germany using a murine Moloney leukemia virusderived retroviral vector encoding the full WASp cDNA. Preliminary data from the first 2 patients 18 months after gene therapy indicate amelioration of the clinical phenotype with correction of thrombocytopenia and resolution of eczema and autoimmunity ${ }^{29}$. Dupre $L$ et al have demonstrated that lentiviral vector encoding a 1.6-kb fragment of the human WAS endogenous promoter (w1.6WLV) is able to successfully restore WASP expression in CD34_HSCs, T cells, B cells, and DCs, and to correct TCR-driven activation in T-cell lines derived from WAS patients ${ }^{30}$. However gene therapy using a lentiviral vector is still in experimental phase in animal model for the treatment of WAS. WAS patient should be immunized except live vaccine because a protective antibody response often obtained.

\section{Conclusion}

We should have high index of suspicion of WAS if any patient presented with bleeding manifestation with history of recurrent sino-pulmonary infection and eczema especially at centres with limited resources in developing country, because early diagnosis and treatment has better prognosis.

\section{References}

1. Aldrich RA, Steinberg AG, Campbell DC.Pedigree demonstrating a sex-linked recessive condition characterized by draining ears, eczematoid dermatitis and bloody diarrhoea. Pediatrics 1954;13:133-39.

2. Inoue R, Kondo N, Kuwabara N, Orii T.Aberrant patterns of immunoglobulin levels in Wiskott-Aldrich syndrome. Scand J Immunol 1955;41:188-93.

3. Mullen CA, Anderson KD, Blases RM. Splenectomy and: or bone marrow transplantation in the management of Wiskott-Aldrich syndrome: long-term follow up of 62 cases. Blood 1993;82:2961-964.

4. Symons M, Derry J, Karlak B, et al. Wiskott- Aldrich syndrome protein, a novel effector for the GTPase CDC42Hs, is implicated in actin polymerization. Cell 1996;84:723-34.

5. Villa A, Notarangelo L, Macchi P, et al. X-linked thrombocytopenia and Wiskott-Aldrich syndrome are allelic diseases with mutations in the WASP gene. Nat Genet 1995;9:414-17.

6. Sullivan $\mathrm{K}$, Mullen $\mathrm{C}$, Blaese $\mathrm{R}$, Winkelstein $\mathrm{J}$. A multi institutional survey of Wiskott-Aldrich syndrome. J Pediatr 1994;125:876-85.

7. Shcherbina A, Rosen F, Remold-O'Donnell E. WASP levels in platelets and lymphocytes of Wiskott-Aldrich syndrome patients correlate with cell dysfunction. J Immunol 1999;163:6314-320.

8. Haddad E, Cramer E, Riviere C, et al. The thrombocytopenia of Wiskott Aldrich syndrome is not related to a defect in proplatelet formation. Blood 1999;94:509-18.

9. Burns S, Cory GO, Vainchenker W, Thrasher AJ. Mechanisms of WASp-mediated hematologic and immunologic disease. Blood 2004;104:3454-462.

10. Imai K, Morio T, Zhu Y, et al. Clinical course of patients with WASP gene mutations. Blood 2004;103:456-64.

11. Trifari S, Sitia G, Aiuti A, et al. Defective Th1 cytokine gene transcription in CD4_ and CD8_ T cells from Wiskott-Aldrich syndrome patients. J Immunol 2006;177:7451-461.

12. Dupuis-Girod S, Medioni J, Haddad E, et al. Autoimmunity in Wiskott-Aldrich syndrome risk factors, clinical features, and outcome in a single center cohort of 55 patients. Pediatrics 2003;111:622-27.

13. Arkwright PD, Abinun M, Cant AJ. Autoimmunity in human primary immunodeficiency diseases. Blood 2002;99:2694-702.

14. Kwan SP, Hagemann TL, Blaese RM, Rosen FS. A high-resolution map of genes, microsatellite markers, and new dinucleotide repeats from UBE1 to the GATA locus in the region Xp11.23. Genomics 1995;29(1):247-52.

15. Snapper SB, Rosen FS. The Wiskott-Aldrich syndrome protein (WASP): roles in signaling 
and cytoskeletal organization. Annu Rev Immunol 1999;17:905-29.

16. Anton IM, Jones GE. WIP: a multifunctional protein involved in actin cytoskeleton regulation. Eur J Cell Biol 2006;85(3-4):295-304.

17. Lum LG, Tubergen DG, Corash L, Blease RM. Splenectomy in the management of the thrombocytopenia of the Wiskott-Aldrich syndrome. N Engl J Med 1980;302:892-96.

18. Antoine C, Muller S, Cant A, et al. Long-term survival and transplantation of haemopoietic stem cells for immunodeficiencies: report of the European experience 1968- 99. Lancet 2003;361:553-60.

19. FilipovichA, Stone J, Tomany S, et al. Impact of donor type on outcome of bone marrow transplantation for Wiskott-Aldrich syndrome: collaborative study of the International Bone Marrow Transplant Registry and the National Marrow Donor Program. Blood 2001;97:1598-603.

20. Kobayashi R, Ariga T, Nonoyama S, et al. Outcome in patients with Wiskott-Aldrich syndrome following stem cell transplantation: an analysis of 57 patients in Japan. Br J Haematol 2006;135:362-66.

21. Ozsahin $H$, Le Deist $F$, Benkerrou $M$, et al. Bone marrow transplantation in 26 patients with WiskottAldrich syndrome from a single center. $J$ Pediatr 1996;129:238-44.

22. Pai SY, Demartiis D, Forino C, et al. Stem cell transplantation for the Wiskott-Aldrich syndrome: a single-center experience confirms efficacy of matched unrelated donor transplantation. Bone Marrow Transplant 2006;38:671-79.

23. Ozsahin $\mathrm{H}$, Cavazzana-Calvo M, Notarangelo LD, et al. Long-term outcome following hematopoietic stem-cell transplantation in Wiskott-Aldrich syndrome: collaborative study of the European Society for Immunodeficiencies and European Group for Blood and Marrow Transplantation. Blood 2008;111:439-45.

24. Mathew LG, Chandy M, Dennison D,et al. Successful bone marrow transplantation in an infant with Wiskott-Aldrich syndrome. Indian Pediatr 1999;36(7):707-10.

25. Wada T, Candotti F. Somatic mosaicism in primary immune deficiencies. Curr Opin Allergy Clin Immunol 2008;8:510-14.

26. Cavazzana-Calvo M, Hacein-Bey S, de Saint Basile $G$, et al. Gene therapy of human severe combined immunodeficiency (SCID)-X1 disease. Science 2000;288:669-72.

27. Ott MG, Schmidt $M$, Schwarzwaelder $K$, et al. Correction of $\mathrm{X}$-linked chronic granulomatous disease by gene therapy, augmented by insertional activation of MDS1-EVI1, PRDM16 or SETBP1.Nat Med 2006;12:401-09.

28. Aiuti A, Cattaneo F, Galimberti F, et al. Long-term safety and efficacy of gene therapy for adenosine deaminase (ADA)-deficient severe combined immunodeficiency. N Engl J Med 2009;360:447-58.

29. Boztug K, Klein C, Avedillo-Diez I, et al. Hematopoitic stem cell gene therapy for Wiskott Aldrich Syndrome. Clinical Experimental Immunology 2008; 154(Suppl.1):12-13(O III-1).

30. Dupre L, Trifari S, Follenzi A, et al. Lentiviral vectormediated gene transfer in $\mathrm{T}$ cells from WiskottAldrich syndrome patients leads to functional correction. Mol Ther 2004;10:903-15.

\section{How to cite this article?}

Kumar MK, Narayan R. Wiskott Aldrich Syndrome, often Missed: A Case Report and Review. J Nep Paedtr Soc 2011;31(2):146-150. 